\title{
NONLINEAR TWO POINT BOUNDARY VALUE PROBLEMS AT RESONANCE WITHOUT LANDESMAN-LAZER CONDITION
}

\author{
R. IANNACCI AND M. N. NKASHAMA
}

(Communicated by George R. Sell)

\begin{abstract}
The purpose of this paper is to study the solvability of a semilinear two-point boundary value problem of resonance type in which the nonlinear perturbation is not (necessarily) required to satisfy Landesman-Lazer condition or the monotonicity assumption. The nonlinearity may be unbounded.
\end{abstract}

\section{INTRODUCTION}

Very recently, the solvability of the semilinear two-point boundary value problem

$$
\begin{aligned}
& u^{\prime \prime}(x)+u(x)+g(x, u(x))=h(x), \\
& u(0)=u(\pi)=0
\end{aligned}
$$

(in which $h \in L^{2}(0, \pi), g:(0, \pi) \times \mathbf{R} \rightarrow \mathbf{R}$ satisfies Carathéodory conditions and grows at most linearly) has been studied by several authors. For some of them, a key sufficient condition, for the existence of at least one solution of equation (1.1), is the so-called Landesman-Lazer condition

$$
\int_{0}^{\pi} g_{-}(x) \sin x d x<\int_{0}^{\pi} h(x) \sin x d x<\int_{0}^{\pi} g_{+}(x) \sin x d x
$$

where $g_{-}(x)=\limsup \operatorname{sum}_{u \rightarrow-\infty} g(x, u)$ and $g_{+}(x)=\liminf _{u \rightarrow \infty} g(x, u)$ (see [1, $2,4,7,8,11,12,16])$. For others, a key condition is that the function $g$ satisfies a monotonicity assumption with respect to the variable $u$ (see $[15,4$, $8,13,16])$.

It is the purpose of this note to provide sufficient conditions for the solvability of the Dirichlet problem (1.1) in which the nonlinearity $g$ is not (necessarily) required to satisfy the condition (1.2) or the monotonicity assumption.

The paper is organized as follows: $\S 2$ is devoted to the main result. At the end of $\S 2$, we illustrate our main theorem by giving an elementary example of

Received by the editors May 27, 1986 and, in revised form, June 1, 1987.

1980 Mathematics Subject Classification (1985 Revision). Primary 34B15, 34C15, 55M25.

Key words and phrases. Semilinear equations at resonance, Dirichlet problem, Neumann problem, Leray-Schauder continuation method, topological degree. 
an unbounded nonlinearity that does satisfy neither condition (1.2) nor monotonicity assumption. In $\S 3$, we are concerned with further result and remarks. In Remark 1, we discuss the relation between our results and previous ones. All of our results are based upon Leray-Schauder continuation method and topological degree [14].

Besides the classical real Lebesgue spaces $L^{p}(0, \pi)$ and the spaces $C^{p}(0, \pi)$ of $p$-times continuously differentiable real valued functions, we shall make use, in what follows, of the Sobolev spaces $H_{0}^{1}(0, \pi)$ and $H^{2}(0, \pi)$ (see e.g. H. Brézis [3] for definitions and properties). In $L^{2}(0, \pi)$, we shall consider the inner product $(u, v)=(2 / \pi) \int_{0}^{\pi} u(x) v(x) d x$ with corresponding modifications for $H^{1}(0, \pi)$ and $H^{2}(0, \pi)$.

\section{THE MAIN RESULT}

Let $h \in L^{2}(0, \pi)$ and $g:(0, \pi) \times \mathbf{R} \rightarrow \mathbf{R}$ be a Carathéodory function i.e. $g(\cdot, u)$ is measurable on $(0, \pi)$ for each $u \in \mathbf{R}, g(x, \cdot)$ is continuous on $\mathbf{R}$ for a.e.. $x \in(0, \pi)$, for any constant $r>0$ there exists a function $\gamma_{r} \in L^{2}(0, \pi)$ such that

$$
|g(x, u)| \leq \gamma_{r}(x)
$$

for a.e. $x \in(0, \pi)$ and all $u \in \mathbf{R}$ with $|u| \leq r$.

Theorem 1. Assume that

$$
g(x, u) u \geq 0
$$

for a.e. $x \in(0, \pi)$ and all $u \in \mathbf{R}$. Moreover, suppose that for all constant $\sigma>0$, there exist a constant $R=R(\sigma)>0$ and a function $b=b(\sigma) \in L^{\infty}(0, \pi)$ such that

$$
|g(x, u)| \leq(\Gamma(x)+\sigma)|u|+b(x)
$$

for a.e. $x \in(0, \pi)$ and all $u \in \mathbf{R}$ with $|u| \geq R$, where $\Gamma \in L^{\infty}(0, \pi)$ is such that, for a.e. $x \in(0, \pi)$

$$
0 \leq \Gamma(x) \leq 3
$$

with $\Gamma(x)<3$ on a subset of $(0, \pi)$ of positive measure.

Then, equation (1.1) has at least one solution for any $h \in L^{2}(0, \pi)$ with

$$
\int_{0}^{\pi} h(x) \sin x d x=0 .
$$

By a solution of equation (1.1) we mean a function $u \in H^{2}(0, \pi) \cap H_{0}^{1}(0, \pi)$ which satisfies the differential equation a.e. To prove Theorem 1 we shall need three useful lemmas stated and proved below.

For each $u \in H_{0}^{1}(0, \pi)$, let us write

$$
u(x)=\bar{u}(x)+\tilde{u}(x)
$$

where $\bar{u}(x)=(2 / \pi)\left(\int_{0}^{\pi} u(x) \sin s d s\right) \sin x$ and $\int_{0}^{\pi} \tilde{u}(x) \sin x d x=0$ so that, with obvious notations $H_{0}^{1}(0, \pi)=\bar{H}_{0}^{1}(0, \pi) \oplus \widetilde{H}_{0}^{1}(0, \pi)$. 
Lemma 1. Let $\Gamma \in L^{\infty}(0, \pi)$ be such that for a.e. $x \in(0, \pi), 0 \leq \Gamma(x) \leq 3$ with $\Gamma(x)<3$ on a subset of $(0, \pi)$ of positive measure. Then, there exists a constant $\delta=\delta(\Gamma)>0$ such that for all $u \in H^{2}(0, \pi) \cap H_{0}^{1}(0, \pi)$, one has

$$
\frac{2}{\pi} \int_{0}^{\pi}\left[u^{\prime \prime}(x)+u(x)+\Gamma(x) u(x)\right](\bar{u}(x)-\tilde{u}(x)) d x \geq \delta|\tilde{u}|_{H^{1}}^{2} .
$$

Proof. Taking into account the orthogonality of $\bar{u}$ and $\tilde{u}$ in $L^{2}(0, \pi)$, one has

$$
\begin{aligned}
& \frac{2}{\pi} \int_{0}^{\pi} {\left[u^{\prime \prime}(x)+u(x)+\Gamma(x) u(x)\right](\bar{u}(x)-\tilde{u}(x)) d x } \\
& \quad=\frac{2}{\pi} \int_{0}^{\pi}\left[\left(\tilde{u}^{\prime}(x)\right)^{2}-(1+\Gamma(x))(\tilde{u}(x))^{2}\right] d x+\frac{2}{\pi} \int_{0}^{\pi} \Gamma(x)(\bar{u}(x))^{2} d x \\
& \quad \geq \frac{2}{\pi} \int_{0}^{\pi}\left[\left(\tilde{u}^{\prime}(x)\right)^{2}-(1+\Gamma(x))(\tilde{u}(x))^{2}\right] d x \equiv D_{\Gamma}(\tilde{u}) .
\end{aligned}
$$

Therefore, using Fourier series and Parseval identity [3], one has $D_{\Gamma}(\tilde{u}) \geq 0$ with equality if and only if $\tilde{u}(x)=A \sin 2 x$ for some $A \in \mathbf{R}$. But then, one gets

$$
0=D_{\Gamma}(\tilde{u})=A^{2} \frac{2}{\pi} \int_{0}^{\pi}(3-\Gamma(x)) \sin ^{2} 2 x d x,
$$

so that by our assumptions, $A=0$ and hence $\tilde{u}=0$.

We claim that there is a constant $\delta=\delta(\Gamma)>0$ such that

$$
D_{\Gamma}(\tilde{u}) \geq \delta|\tilde{u}|_{H^{1}}^{2} .
$$

Assume that the claim is not true. Then, we can find a sequence $\left(\tilde{u}_{n}\right) \subset$ $\tilde{H}_{0}^{1}(0, \pi)$ and $\tilde{u} \in \tilde{H}_{0}^{1}(0, \pi)$ such that, by using the compact embedding of $\tilde{H}_{0}^{1}(0, \pi)$ into $C(0, \pi)$ and passing to a subsequence if necessary [3],

$$
\left|\tilde{u}_{n}\right|_{H^{1}}=1, \quad \tilde{u}_{n} \rightarrow \tilde{u} \quad \text { in } C(0, \pi), \quad \tilde{u}_{n} \rightarrow \tilde{u} \quad \text { in } \tilde{H}_{0}^{1}(0, \pi),
$$

and

$$
0 \leq D_{\Gamma}\left(\tilde{u}_{n}\right) \leq 1 / n, \quad n \in \mathbf{N}^{*} .
$$

Since $\tilde{H}_{0}^{1}(0, \pi)$ is a Hilbert space and $\tilde{u}_{n}$ converges weakly to $\tilde{u}$ in $\tilde{H}_{0}^{1}(0, \pi)$, one has [3]

$$
|\tilde{u}|_{H^{1}}^{2} \leq \liminf _{n \rightarrow \infty}\left|\tilde{u}_{n}\right|_{H^{1}}^{2}
$$

By (2.7) and (2.8), we obtain, for $n \rightarrow \infty$,

$$
\left|\tilde{u}_{n}\right|_{H^{1}}^{2} \rightarrow \frac{2}{\pi} \int_{0}^{\pi}(1+\Gamma(x))(\tilde{u}(x))^{2} d x
$$

and hence

$$
|\tilde{u}|_{H^{1}}^{2} \leq \frac{2}{\pi} \int_{0}^{\pi}(1+\Gamma(x))(\tilde{u}(x))^{2} d x, \quad \text { i.e., } \quad D_{\Gamma}(\tilde{u}) \leq 0 .
$$

By the first part of the proof, $\tilde{u}=0$, so that, by (2.9), $\left|\tilde{u}_{n}\right|_{H^{1}} \rightarrow 0$, a contradiction with the first equality in (2.7) and the proof is complete. 
Lemma 2. Let $\Gamma$ be like in Lemma 1 and $\delta>0$ be associated with $\Gamma$ by that Lemma. Let $\sigma>0$. Then, for all $p \in L^{\infty}(0, \pi)$ satisfying

$$
0 \leq p(x) \leq \Gamma(x)+\sigma
$$

a.e. on $(0, \pi)$ and all $u \in H^{2}(0, \pi) \cap H_{0}^{1}(0, \pi)$ one has

$$
\frac{2}{\pi} \int_{0}^{\pi}\left[u^{\prime \prime}(x)+u(x)+p(x) u(x)\right](\bar{u}(x)-\tilde{u}(x)) d x \geq(\delta-\sigma)|\tilde{u}|_{H^{1}}^{2} .
$$

Proof. Using computations of Lemma 1, we obtain

$$
\begin{aligned}
& \frac{2}{\pi} \int_{0}^{\pi}\left[u^{\prime \prime}(x)+u(x)+p(x) u(x)\right](\bar{u}(x)-\tilde{u}(x)) d x \\
& \quad \geq \frac{2}{\pi} \int_{0}^{\pi}\left[\left(\tilde{u}^{\prime}(x)\right)^{2}-(1+p(x))(\tilde{u}(x))^{2}\right] d x \equiv D_{p}(\tilde{u}) .
\end{aligned}
$$

Therefore, by the second inequality in (2.10), one has

$$
D_{p}(\tilde{u}) \geq D_{\Gamma}(\tilde{u})-\sigma \frac{2}{\pi} \int_{0}^{\pi}(\tilde{u}(x))^{2} d x .
$$

So that, using again Fourier series, Parseval identity and Lemma 1, one deduces that $D_{p}(\tilde{u}) \geq(\delta-\sigma)|\tilde{u}|_{H^{1}}^{2}$ and the proof is complete.

Let us consider the subspace $H_{\pi}^{2}(0, \pi) \subset H^{2}(0, \pi)$ defined by

$$
H_{\pi}^{2}(0, \pi)=H^{2}(0, \pi) \cap H_{0}^{1}(0, \pi) \equiv\left\{u \in H^{2}(0, \pi): u(0)=u(\pi)=0\right\} .
$$

Since $H^{2}(0, \pi)$ is continuously embedded into $C(0, \pi)$, it follows immediately that $H_{\pi}^{2}(0, \pi)$ is a closed subspace of the Hilbert space $H^{2}(0, \pi)$ so that it is also a Hilbert space with respect to the inner product of $H^{2}(0, \pi)$ [3].

Lemma 3. Let $q \in(0,3)$ be fixed. Then, there exists a constant $\eta>0$ such that for all $u \in H_{\pi}^{2}(0, \pi)$, one has

$$
\left|u^{\prime \prime}+u+q u\right|_{L^{2}} \geq \eta|u|_{H^{2}} \text {. }
$$

Proof. By the theory of linear second order differential equations [1, 3, 15], the operator $E: H_{\pi}^{2}(0, \pi) \rightarrow L^{2}(0, \pi)$ defined by $E u=u^{\prime \prime}+u+q u$ is one-toone, onto and obviously continuous. It follows that $E^{-1}: L^{2}(0, \pi) \rightarrow H_{\pi}^{2}(0, \pi)$ is linear and continuous [3, p. 19]. Taking $\eta \leq\left(1 /\left|E^{-1}\right|\right)$, the proof is complete.

Proof of Theorem 1. Let $\delta>0$ be associated to the function $\Gamma$ by Lemma 1 . Then, by assumption (2.3), there exist $R(\delta)>0$ and $b=b(\delta) \in L^{\infty}(0, \pi)$ such that

$$
|g(x, u)| \leq(\Gamma(x)+\delta / 4)|u|+b(x)
$$

for a.e. $x \in(0, \pi)$ and all $u \in \mathbf{R}$ with $|u| \geq R$. Without loss of generality, we can choose $R$ such that $b(x) /|u|<\delta / 4$ for a.e. $x \in(0, \pi)$ and all $u \in \mathbf{R}$ with 
$|u| \geq R$. Let us define $\tilde{\gamma}:(0, \pi) \times \mathbf{R} \rightarrow \mathbf{R}$ by

$$
\tilde{\gamma}(x, u)= \begin{cases}u^{-1} g(x, u) & \text { for }|u| \geq R, \\ R^{-1} g(x, R)(u / R)+(1-u / R) \Gamma(x) & \text { for } 0 \leq u \leq R, \\ R^{-1} g(x,-R)(u / R)+(1+u / R) \Gamma(x) & \text { for }-R \leq u \leq 0 .\end{cases}
$$

Then, by assumption (2.2) and relation (2.12), we have

$$
0 \leq \tilde{\gamma}(x, u) \leq \Gamma(x)+\delta / 2
$$

for a.e. $x \in(0, \pi)$ and all $u \in \mathbf{R}$. Moreover the function $\tilde{\gamma}(x, u) u$ satisfies Carathéodory conditions and $f:(0, \pi) \times \mathbf{R} \rightarrow \mathbf{R}$ defined by

$$
f(x, u)=g(x, u)-\tilde{\gamma}(x, u) u
$$

is such that, for a.e. $x \in(0, \pi)$ and all $u \in \mathbf{R}$,

$$
|f(x, u)| \leq \nu(x)
$$

for some $\nu \in L^{2}(0, \pi)$ depending only on $\Gamma$ and $\gamma_{R}$ given by (2.1).

Therefore, equation (1.1) is equivalent to

$$
\begin{gathered}
u^{\prime \prime}(x)+u(x)+\tilde{\gamma}(x, u(x)) u(x)+f(x, u(x))=h(x), \\
u(0)=u(\pi)=0 .
\end{gathered}
$$

To prove that equation (2.16) has at least one solution, it suffices, according to the Leray-Schauder continuation method [14], to show that the possible solutions of the family of equations

$$
\begin{gathered}
u^{\prime \prime}+u+(1-\lambda) q u+\lambda \tilde{\gamma}(x, u) u+\lambda f(x, u)=\lambda h, \\
u(0)=u(\pi)=0, \quad \lambda \in[0,1)
\end{gathered}
$$

(in which $q \in(0,3)$ with $q<\delta / 2, q$ fixed) are a priori bounded in $C^{1}(0, \pi)$ independently of $\lambda \in[0,1)$. Notice that, by inequalities $(2.13)$, one has

$$
0 \leq(1-\lambda) q+\lambda \tilde{\gamma}(x, u) \leq \Gamma(x)+\delta / 2
$$

for a.e. $x \in(0, \pi)$ and all $u \in \mathbf{R}$.

It is clear that for $\lambda=0$, equation (2.17) has only the trivial solution. Now, if $u \in H_{\pi}^{2}(0, \pi)$ is a solution of $(2.17)$ for some $\lambda \in(0,1)$, then using Lemma 2 and Cauchy-Schwarz inequality [3], we get

$$
\begin{aligned}
0= & \frac{2}{\pi} \int_{0}^{\pi}(\bar{u}(x)-\tilde{u}(x))\left(u^{\prime \prime}(x)+u(x)\right)+[(1-\lambda) q+\lambda \tilde{\gamma}(x, u(x))] u(x) d x \\
& +\frac{2}{\pi} \int_{0}^{\pi}(\bar{u}(x)-\tilde{u}(x))(\lambda f(x, u(x))-\lambda h(x)) d x \\
\geq & (\delta / 2)|\tilde{u}|_{H^{1}}^{2}-\left(|\bar{u}|_{L^{2}}+|\tilde{u}|_{L^{2}}\right)\left(|f|_{L^{2}}+|h|_{L^{2}}\right),
\end{aligned}
$$

so that, by the compact embedding of $H^{1}(0, \pi)$ into $L^{2}(0, \pi)$ and relation (2.15), we deduce

$$
0 \geq(\delta / 2)|\tilde{u}|_{H^{1}}^{2}-\beta\left(|\tilde{u}|_{H^{1}}+|\bar{u}|_{H^{1}}\right)
$$


for some constant $\beta>0$ depending only on $\nu$ and $h$ (but not on $u$ or $\lambda$ ). Taking $\alpha=\beta(\delta)^{-1}$, we get

$$
|\tilde{u}|_{H^{1}} \leq \alpha+\left(\alpha^{2}+2 \alpha|\bar{u}|_{H^{1}}\right)^{1 / 2}
$$

We claim that there exists a constant $\rho>0$ such that

$$
|u|_{C^{1}}<\rho
$$

for all solutions $u \in H_{\pi}^{2}(0, \pi)$ of equation (2.17) ( $\rho$ independent of $u$ and $\lambda)$. If we assume that the claim is false, then there exist sequences $\left(\lambda_{n}\right)$ in the open interval $(0,1)$, and $\left(u_{n}\right)$ in $H_{\pi}^{2}(0, \pi)$ with $\left|u_{n}\right|_{C^{1}} \geq n$ for all $n \in \mathbf{N}^{*}$ such that

$$
\begin{gathered}
u_{n}^{\prime \prime}+u_{n}+\left(1-\lambda_{n}\right) q u_{n}+\lambda_{n} g\left(x, u_{n}\right)=\lambda_{n} h, \\
u_{n}(0)=u_{n}(\pi)=0 .
\end{gathered}
$$

Setting $v_{n}=\left(u_{n} /\left|u_{n}\right|_{C^{1}}\right)$, we have

$$
\begin{gathered}
v_{n}^{\prime \prime}+v_{n}+q v_{n}=\lambda_{n}\left(h /\left|u_{n}\right|_{C^{1}}\right)+\lambda_{n} q v_{n}-\lambda_{n}\left(g\left(x, u_{n}(x)\right) /\left|u_{n}\right|_{C^{1}}\right), \\
v_{n}(0)=v_{n}(\pi)=0
\end{gathered}
$$

or equivalently

$$
E v_{n}=\lambda_{n}\left(h /\left|u_{n}\right|_{C^{1}}\right)+\lambda_{n} q v_{n}-\lambda_{n}\left(g\left(x, u_{n}\right) /\left|u_{n}\right|_{C^{1}}\right)
$$

where $E: H_{\pi}^{2}(0, \pi) \subset C^{1}(0, \pi) \rightarrow L^{2}(0, \pi)$ is defined by $E v=v^{\prime \prime}+v+q v$.

According to Lemma 3 and the compact embedding of $H^{2}(0, \pi)$ into $C^{1}(0, \pi)$ [3], $E$ is invertible and $E^{-1}$ is compact (completely continuous) as an operator from $L^{2}(0, \pi)$ into $C^{1}(0, \pi)$. On the other hand, by relation (2.1) and the growth condition (2.3), it follows that there exists a function $c \in L^{2}(0, \pi)$ depending only on $R=R(\delta)>0$ such that

$$
|g(x, u)| \leq(\Gamma(x)+\delta / 2)|u|+b(x)+c(x)
$$

for a.e. $x \in(0, \pi)$ and all $u \in \mathbf{R}$. So that, the sequence $\left(g\left(x, u_{n}\right) /\left|u_{n}\right|_{C^{1}}\right)$ is bounded in $L^{2}(0, \pi)$. Hence, the right-hand member of equality $(2.24)$ is bounded in $L^{2}(0, \pi)$ independently of $n$. Therefore, writing equation (2.24) in the equivalent form

$$
v_{n}=E^{-1}\left[\lambda_{n}\left(h /\left|u_{n}\right|_{C^{1}}\right)+\lambda_{n} q v_{n}-\lambda_{n}\left(g\left(x, u_{n}\right) /\left|u_{n}\right|_{C^{1}}\right)\right]
$$

and using the compactness of $E^{-1}: L^{2}(0, \pi) \rightarrow C^{1}(0, \pi)$ we can assume (going if necessary to a subsequence relabeled $\left.\left(v_{n}\right)\right)$ that there exists $v \in C^{1}(0, \pi)$ such that $v_{n} \rightarrow v$ in $C^{1}(0, \pi)$ for $n \rightarrow \infty,|v|_{C^{1}}=1$ and $v(0)=v(\pi)=0$.

On the other hand, using inequality (2.19) or (2.20), one deduces immediately that $\tilde{v}_{n} \rightarrow 0$ in $H^{1}(0, \pi)$ for $n \rightarrow \infty$. Therefore $v \in \bar{H}_{0}^{1}(0, \pi)$ i.e. $v(x)=$ $A \sin x$ for some $A \in \mathbf{R}$. Since $|v|_{C^{1}}=1$, one has $v(x)= \pm \sin x$. In what follows, we shall suppose that $v(x)=\sin x$ (the case $v(x)=-\sin x$ is treated in a similar way). 
Now, using the fact that $v_{n}(0)=v_{n}(\pi)=0, v_{n} \rightarrow v$ in $C^{1}(0, \pi)$ with $v(x)=\sin x, v_{n}^{\prime}(0) \rightarrow 1$ and $v_{n}^{\prime}(\pi) \rightarrow-1$ for $n \rightarrow \infty$, we have that there exists $n_{0} \in \mathbf{N}^{*}$ such that, for $n \geq n_{0}, v_{n}(x)>0$ on $(0, \pi)$. So that, for $n \geq n_{0}$,

$$
u_{n}(x)>0 \quad \text { on }(0, \pi), \quad u_{n}(0)=u_{n}(\pi)=0 .
$$

Writing $v_{n}=\bar{v}_{n}+\tilde{v}_{n}$, we have $\bar{v}_{n}(x)=K_{n} \sin x$ with $K_{n} \rightarrow 1$ for $n \rightarrow \infty$.

Let us come back to equation (2.23). Taking the inner product (in $L^{2}(0, \pi)$ ) of (2.23) with $\bar{v}_{n}$, remarking that $\lambda_{n} \in(0,1)$ and considering assumption (2.5), we deduce that

$$
\left(\lambda_{n} /\left|u_{n}\right|_{C^{1}}\right) \int_{0}^{\pi} g\left(x, u_{n}(x)\right) \bar{v}_{n}(x) d x<0
$$

for all $n$ sufficiently large, so $\int_{0}^{\pi} g\left(x, u_{n}(x)\right) \sin x d x<0$. This is a contradiction, since by (2.26) and assumption (2.2), one has $g\left(x, u_{n}(x)\right) \sin x \geq 0$ on $(0, \pi)$ for $n \geq n_{0}$ and the proof is complete.

Example. Let us consider equation (1.1) in which $g:(0, \pi) \times \mathbf{R} \rightarrow \mathbf{R}$ is defined by

$$
g(x, u)=\Gamma(x) u \sin ^{2} u
$$

where $\Gamma(x)=0$ for $x \in(0, \pi / 2)$ and $\Gamma(x)=3$ for $x \in[\pi / 2, \pi)$.

It is immediately seen that all assumptions of Theorem 1 are fulfilled. So that equation (1.1) has at least one solution for any $h \in L^{2}(0, \pi)$ with

$$
\int_{0}^{\pi} h(x) \sin x d x=0 .
$$

Obviously $g(x, \cdot)$ is not monotone on $[\pi / 2, \pi)$. However, since $g(x, \pm k \pi)$ $=0$ for all $x \in(0, \pi)$ and all $k \in \mathbf{N}$, we have $\limsup _{u \rightarrow-\infty} g(x, u)=$ $\liminf _{u \rightarrow \infty} g(x, u)=0$. Hence, the nonlinearity (2.28) does not satisfy the Landesman-Lazer condition (1.2). Notice that $g$ is unbounded.

\section{FURTHER RESULT AND REMARKS}

Following step by step the approach used in $\S 2$ with obvious modifications in notations, one has

Theorem 2. Let $g$ be a Carathéodory function. Assume that there exists a constant $r>0$ such that

$$
g(x, u) u \geq 0
$$

for a.e. $x \in(0, \pi)$ and all $u \in \mathbf{R}$ with $|u| \geq r$. Moreover, suppose that assumption (2.3) is fulfilled, where $\Gamma \in L^{\infty}(0, \pi)$ is such that, for a.e. $x \in(0, \pi)$ (3.2) $0 \leq \Gamma(x) \leq 1$ with $\Gamma(x)<1$ on a subset of $(0, \pi)$ of positive measure. Then, the Neumann problem

$$
\begin{gathered}
u^{\prime \prime}(x)+g(x, u(x))=h(x), \\
u^{\prime}(0)=u^{\prime}(\pi)=0
\end{gathered}
$$


has at least one solution for any $h \in L^{2}(0, \pi)$ with

$$
\int_{0}^{\pi} h(x) d x=0
$$

Notice that, in this case, relation (2.26) reduces to

$$
u_{n}(x) \geq r \quad \text { on }[0, \pi], \quad u_{n}^{\prime}(0)=u_{n}^{\prime}(\pi)=0 .
$$

Remark 1. Theorem 1 is a strong improvement of main results contained in $[6,9]$ since they assumed $h \in L^{\infty}(0, \pi)$ satisfying $(2.5)$ and $g(x, u) \equiv g(u)$ a bounded continuous function. Notice that assumptions $(2.3)-(2.4)$ are automatically satisfied when $g$ is bounded.

In [10, Theorem 4] (resp. Theorem 5), there is a result similar to our Theorem 1 (resp. Theorem 2) with $h \in L^{\infty}(0, \pi), g(x, u)$ a continuous function and $\Gamma(x) \leq q$ for all $x \in(0, \pi)$ where $q$ is a constant such that $q<3$ (resp. $q<1$ ). Obviously those results are a special case of ours (see e.g. the example of $\S 2$ ). Let us mention here that contrary to what the author is affirming in [10], his abstract results do not apply to the Dirichlet problem. More precisely, we shall give an example of a nonlinearity $g$ which satisfies all assumptions of Theorems 1 and 4 of [10], but the assumption (iii) of his abstract results is not fulfilled (see [10, p. 928]). Therefore, it seems to us that the proof proposed in $[10]$ is lacunar. Indeed, let us consider

$$
g(x, u)= \begin{cases}0 & \text { if } x \in(0, \pi) \text { and } u \geq 0, \\ u & \text { if } x \in(0, \pi) \text { and } u \leq 0 .\end{cases}
$$

Obviously all assumptions of Theorems 1 and 4 of [10] are satisfied since $g(x, u) u \geq 0$ for all $x \in(0, \pi)$, all $u \in \mathbf{R}$ and lim $\sup _{|u| \rightarrow \infty} g(x, u) / u=1<3$.

On the other hand, using the definitions of the operators $Q, P, N$ given in the proofs of Theorems 1 and 4 of [10] and considering our notations of $\S 2$, the assumption (iii) of [10, p. 928], is equivalent to

For $u \in L^{\infty}(0, \pi)$ with

$$
\int_{0}^{\pi}\left[\left(\int_{0}^{\pi} g(s, u(s)) \sin s d s\right)(\sin x) \bar{u}(x) d x\right]<0, \quad|\tilde{u}|_{L^{\infty}} \leq \text { constant }
$$

implies that there exists a constant $C$ such that $|\bar{u}|_{L^{\infty}} \leq C$.

We shall now exhibit a sequence $\left(u_{n}\right)$ in $H_{\pi}^{2}(0, \pi) \equiv H^{2}(0, \pi) \cap H_{0}^{1}(0, \pi) \subset$ $L^{\infty}(0, \pi)$ which shows that the nonlinearity given by (3.6) does not fulfill the assumption (3.7). Indeed (for $n \in \mathbf{N}^{*}$ ), let us consider the sequence

$$
u_{n}(x)=n \sin x-\sin 4 n^{2} x, \quad x \in[0, \pi] .
$$

We have immediately that $u_{n}(0)=0$ and $u_{n}^{\prime}(0)=n-4 n^{2}<0$. Therefore there exists $x_{n} \in(0, \pi]$ such that

$$
u_{n}(x)<0 \quad \text { on }\left(0, x_{n}\right), \quad u_{n}(0)=u_{n}\left(x_{n}\right)=0 .
$$

Let us define the subsets $I_{n}=\left\{x \in(0, \pi): u_{n}(x)<0\right\}$. It follows from (3.9) that $I_{n}$ is of positive measure i.e.

$$
\operatorname{mes}\left(I_{n}\right)>0 \quad \text { for each } n \in \mathbf{N}^{*} .
$$


Observing that $\bar{u}_{n}(x)=n \sin x$, using the construction of $g$ in (3.6) and the property (3.10), one has that for each $n \in \mathbf{N}^{*}$,

$$
\int_{0}^{\pi} g\left(s, u_{n}(s)\right) \sin s d s=\int_{u_{n}(s) \leq 0} u_{n}(s) \sin s d s=\int_{I_{n}} u_{n}(s) \sin s d s<0 .
$$

On the other hand, since $\tilde{u}_{n}(x)=-\sin 4 n^{2} x$, one has $\left|\tilde{u}_{n}\right|_{L^{\infty}}=1$, but $\left|\bar{u}_{n}\right|_{L^{\infty}}=n$, so that $\left|\bar{u}_{n}\right|_{L^{\infty}} \rightarrow \infty$ for $n \rightarrow \infty$. Thus assumption (3.7) is not satisfied. Notice that $\left|\tilde{u}_{n}^{\prime}\right|_{L^{\infty}}=4 n^{2}$.

Our approach corrects the above lacunae and gives a more general result. Finally, let us mention that the sequence (3.8) cannot be solutions of some homotopy given in (2.17) since they do not satisfy the inequality (2.20) that follows from Lemma 2.

According to the above discussion, we wish to emphasize that our Theorem 1 may be considered as a first result dealing with the solvability of the nonlinear Dirichlet problem for the unbounded case in which there is neither LandesmanLazer condition nor monotonicity assumption.

For others results concerning the solvability of equation (1.1) in which the nonlinearity is still bounded without condition (1.2), we refer to [5, 13].

Remark 2. In Theorem 1, the sign condition (2.2) cannot be replaced by (3.1) which is weaker, we refer to the counterexample given in [6, p. 633]. On the other hand, if inequalities (2.2) and (3.1) are reversed, the growth conditions (2.3)-(2.4) and (2.3)-(3.2) are not needed; we refer to References [4] in [1] and [9] in [5]. Moreover, since the problem $u^{\prime \prime}+u+3 u=\sin 2 x, u(0)=u(\pi)=0$ (resp. $u^{\prime \prime}+u=\cos x, u^{\prime}(0)=u^{\prime}(\pi)=0$ ) has no solution, the condition $\Gamma(x)<3$ (resp. $\Gamma(x)<1$ ) on a subset of $(0, \pi)$ of positive measure is, in some sense, sharp.

\section{REFERENCES}

1. S. Ahmad, A resonance problem in which the nonlinearity may grow linearly, Proc. Amer. Math. Soc. 92 (1984), 381-384.

2. H. Berestycki and D. G. De Figueiredo, Double resonance in semi-linear elliptic problems, Comm. Partial Differential Equations 6 (1981), 91-120.

3. H. Brézis, Analyse fonctionnelle, théorie et applications, Masson, Paris, 1983.

4. L. Cesari and R. Kannan, Existence of solutions of a nonlinear differential equation, Proc. Amer. Math. Soc. 88 (1983), 605-613.

5. __, Qualitative study of a class of nonlinear boundary value problem at resonance, J. Differential Equations 56 (1985), 63-81.

6. D. G. De Figueiredo and W. M. Ni, Perturbations of second order linear elliptic problems by nonlinearities without Landesman-Lazer condition, Nonlinear Anal. 3 (1979), 629-634.

7. P. Drábek, On the resonance problem with nonlinearity which has arbitrary linear growth, J. Math. Anal. Appl. 127 (1987), 435-442.

8. S. Fucik, Surjectivity of operators involving linear noninvertible part and nonlinear compact perturbation, Funkcial. Ekvac. 17 (1974), 73-83.

9. J. V. A. Gonçalves, On bounded nonlinear perturbations of an elliptic equation at resonance, Nonlinear Anal. 5 (1981), 57-60. 
10. C. P. Gupta, Perturbations of second order linear elliptic problems by unbounded nonlinearities, Nonlinear Anal. 6 (1982), 919-933.

11. R. Iannacci and M. N. Nkashama, Nonlinear boundary value problems at resonance, Nonlinear Anal. 11 (1987), 455-473.

12. __ Unbounded perturbations of forced second order ordinary differential equations at resonance, J. Differential Equations 69 (1987), 289-309.

13. R. Kannan, J. J. Nieto and M. B. Ray, A class of nonlinear boundary value problems without Landesman-Lazer condition, J. Math. Anal. Appl. 105 (1985), 1-11.

14. N. G. Lloyd, Degree theory, Cambridge Univ. Press, 1978.

15. J. Mawhin, J. R. Ward and M. Willem, Necessary and sufficient conditions for the solvability of a nonlinear two-point boundary value problem, Proc. Amer. Math. Soc. 93 (1985), 667-674.

16. M. Schechter, J. Shapiro and M. Snow, Solution of the nonlinear problem $A u=N(u)$ in $a$ Banach Space, Trans. Amer. Math. Soc. 241 (1978), 69-78.

Dipartimento di Metodi e Modelli Matematici per le Scienze Applicate, Università "LA Sapienza", Via A. Scarpa, 10,00161 Roma, Italy

Department of Mathematical Sciences, Memphis State University, Memphis, TENNESSEE 38152 